\title{
Fracture spacing in layered materials and pattern transition from parallel to polygonal fractures
}

\author{
C. A. Tang, ${ }^{1, *}$ Y. B. Zhang, ${ }^{2}$ Z. Z. Liang, ${ }^{1}$ T. Xu, ${ }^{3}$ L. G. Tham, ${ }^{4}$ P.-A. Lindqvist, ${ }^{5}$ S. Q. Kou, ${ }^{5}$ and H. Y. Liu \\ ${ }^{1}$ Department of Engineering Mechanics, Dalian University of Technology, Dalian, People's Republic of China \\ ${ }^{2}$ Center for Rock Instability and Seismicity Research, Northeastern University, Shenyang, People's Republic of China \\ ${ }^{3}$ Center for Material Failure Modeling Research, Dalian University, Dalian, People's Republic of China \\ ${ }^{4}$ Rock Engineering Research Center, The University of Hong Kong, Hong Kong, People's Republic of China \\ ${ }^{5}$ Rock Engineering Division, Lulea University of Technology, Lulea, Sweden
}

(Received 28 July 2005; revised manuscript received 13 December 2005; published 22 May 2006)

\begin{abstract}
We perform three-dimensional simulations of fracture growth in a three-layered plate model with an embedded heterogeneous layer under horizontal biaxial stretch (representing stretch from directional to isotropic) by the finite element approach. The fractures develop under a quasistatical, slowly increasing biaxial strain. The material inhomogeneities are accounted for by assigning each element a failure threshold that is defined by a given statistical distribution. A universal scale law of fracture spacing to biaxial strain in terms of principal stress ratio is well demonstrated in a three-dimensional fashion. The numerically obtained fracture patterns show a continuous pattern transition from parallel fractures, laddering fracture to polygonal fractures, which depends strongly on the far-field loading conditions in terms of principal stress ratio $\left(\lambda=\sigma_{2} / \sigma_{1}\right)$, from uniaxial $(\lambda=0)$, anisotropic $(0<\lambda<1)$ to isotropic stretch $(\lambda=1)$. We find that, except for further opening of existing fractures after they are well-developed (saturation), new fractures may also initiate and propagate along the interface between layers, which may serve as another mechanism to accommodate additional strain for fracture saturated layers.
\end{abstract}

DOI: 10.1103/PhysRevE.73.056120

PACS number(s): 46.50.+a, 46.55.+d

\section{INTRODUCTION}

When natural and engineered systems are subjected to shrinkage-driven by cooling or drying - the resulting stresses may lead to formation of fractures [1-18]. Experimental studies of this phenomenon and the fracture spacing theory $[10,14]$ show that the spacing between fractures initially decreases as extensional strain increases in the direction perpendicular to the fractures. At a certain ratio of spacing to layer thickness, however, no new fractures form and the additional strain is accommodated by the further opening of existing fractures: the spacing then simply scales with layer thickness, which is called fracture saturation $[10,14]$.

In field or laboratory observations, two kinds of fracture patterns are commonly observed-parallel fractures [Fig. 1(a)] and polygonal fractures [Fig. 1(b)]. A parallel fracture pattern occurs in layered materials usually under a mechanical layer-parallel stretching force, i.e., directional extension or biaxial stretch with one of the principal stresses much greater than the other. A polygonal fracture pattern is often observed in surface layered materials under cooling or shrinking induced isotropic stretch in all layer-parallel directions. Examples for the polygonal fracture pattern in geosciences are desiccation fractures in dried-out mud flats or in a turtle cracking rock [Fig. 1(b)], permafrost, densificationinduced shattering of the upper crust $[19,20]$. A keen area of research in engineered systems is to look at the fracture patterns in composite materials. When thin glass strips are exposed to uniaxial tensile strain, such as that caused by thermal gradient [21] or drying of thin colloidal suspensions [22], uniformly spaced fractures form parallel to the direc-

*Corresponding author. Electronic address: tca@mail.neu.edu.cn tion of the temperature or moisture gradient. Alternatively, for a brittle film that is attached to a substrate subjected to biaxial tension, the formed fractures divide the film into a series of polygonal shaped islands in a pattern that is often seen in drying mud [7]. The surface cooling fracturing, an ancient technique to decorate china with surface fracture network, is another classic example of this polygonal fracture phenomenon.

In a recent experimental study, Shorlin et al. [28] observed another mode of fracture pattern that they refer to as "laddering." In their investigation, two types of experiments were performed: "isotropic drying," in which the entire layer was dried uniformly; and "directional drying," in which the layer was dried from one end. They observed a change in the way in which fragmentation occurred as the drying method in the system was varied: for isotropic drying, more uniformed polygons formed. For directional drying, polygons did not formed in regular shape, but rather formed by laddering. In this process, fractures propagate more or less parallel to each other. As the layer behind the moving fracture tips dries further, perpendicular fractures form, joining two of the parallel fractures like the rungs of a ladder. This process is dominant in the directional drying case, and clearly involves a local directionality of the drying process in an essential way. Since the directional drying will result in a anisotropic stretch in the tested layer, we believe that this laddering fracture pattern should relate to another loading condition, that is, between the isotropic stretch and the directional or uniaxial stretch. In this paper, using a fracture modeling approach based on the finite element method, we could examine these different fracture patterns in a more controlled way. We find that the numerically obtained fracture patterns show a continuous pattern transition from parallel fractures, laddering fracture to polygonal fractures, which depends strongly on the far-field loading conditions in terms of prin- 

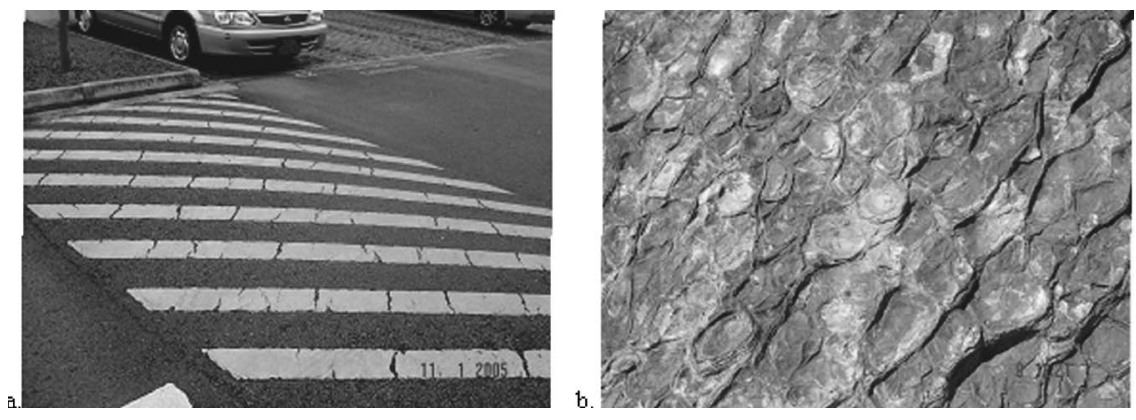

FIG. 1. Examples of a parallel fracture pattern in a road surface (a) and polygonal fracture pattern in a turtle cracking rock taking shape in the Sinian period of 600 million years $(\mathrm{b})$. cipal stress ratio $\left(\lambda=\sigma_{2} / \sigma_{1}\right)$, from uniaxial $(\lambda=0)$, biaxial $(0<\lambda<1)$ to isotropic stretch $(\lambda=1)$.

Modeling the fracture patterns in a rock mass is generally a three-dimensional problem (Fig. 2) and requires a fracture analysis approach. However, most of the present studies can only address the common, albeit particular, cases of parallel vertical joints that are perpendicular to tabular layered rocks exposed in a vertical section, which is almost perpendicular to the strike of the studied joint set $[1,4,5,9-11,13]$. In addition, almost all numerical models of fracture spacing are based on the stress analysis approach $[13,14]$, and a few of the existing models can adequately reproduce the evolution process of fracture nucleation, propagation, infilling, and saturation, as observed experimentally.

The remainder of this paper is organized as follows. In Sec. II, we describe our numerical method. In Sec. III we discuss the numerical results, first on the typical result of the fracture pattern evolution, taking isotropic loading as a example, and then on the fracture patterns under nine different loading conditions representing loads from uniaxial to biaxial stretch. Sections IV and V contain a discussion and a brief conclusion.

\section{NUMERICAL METHOD AND THE THREE-LAYER MODEL}

Here we consider a three-layered model (Fig. 3) that fails under a quasistatical, slowly increasing biaxial strain (induced, for example, by temperature changes, desiccation, or mechanical deformations). The model is consisting of 1.6 million elements $(200 \times 200 \times 40)$. In order to limit the frac-

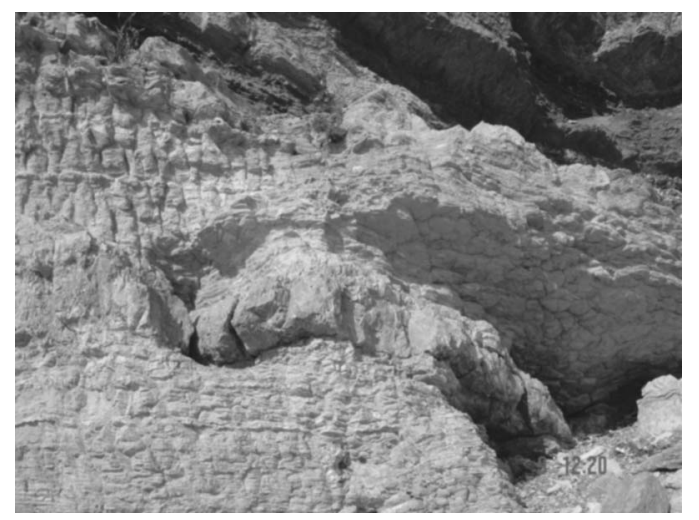

FIG. 2. Example of polygonal fracture pattern showing the three-dimensional fashion. tures that occur in the central layer, the strength of the upper and bottom layers are set to be many times higher than the central layer. The value at which a particular element breaks is random, but fixed at the start of the fragmentation process (i.e., the disorder is quenched). The probability distribution (PD) of breakdown thresholds is a material property and is known from the start $[23,24]$. We account for this local randomness by assigning to each element a failure threshold defined by the Weibull PD, in which two parameters to control the distribution characteristics are introduced: the scale parameter $u_{0}$ that relates to the average of element parameter and the parameter $m$ that defines the shape of the distribution function. The parameter $m$ defines the degree of material homogeneity and is called the homogeneity index.

Initially, elements are considered to be elastic; their elastic properties are defined by Young's modulus and Poisson's ratio. The stress-strain curve of each element is considered to be linear elastic until the given damage threshold is attained. We choose the maximum tensile stress criterion and the Mohr-Coulomb criterion, respectively, as the damage thresholds. The tensile criterion is used primarily to determine whether or not the element is damaged in tensile mode. If the element is not damaged in tensile mode, then Mohr-Coulomb criterion is used to judge whether the element damage occurs in shear modes.

When the element is under uniaxial tension, the constitutive relationship is illustrated in the left part of Fig. 4. It

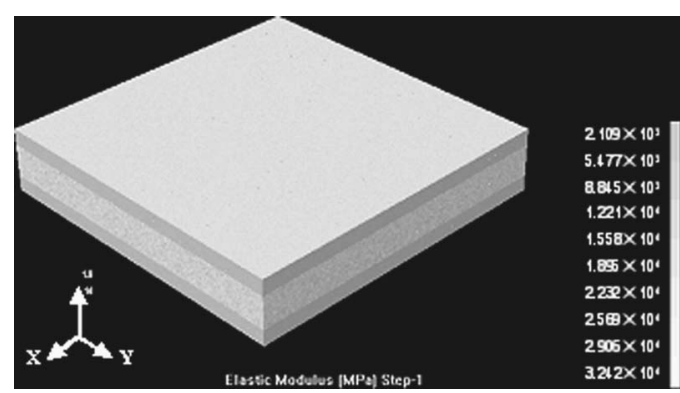

FIG. 3. FEM model with a heterogeneous central embedded layer bonded to top and bottom layers. The model consists of 1.6 million elements $(200 \times 200 \times 40)$. The horizontal plane is defined as $x-y$ plane and $z$ is the vertical direction. We fix the whole bottom boundary in the vertical $z$ direction, and the top boundary is free to displace as necessary. We use a constant displacement increment in the $x$ direction along the left and right boundary, and use another increment in the $y$ direction along front and back boundary. We define $\lambda$ as the loading ratio of the displacement in the $x$ direction to the displacement in the $y$ direction in order to model varies of principal stress ratio. 


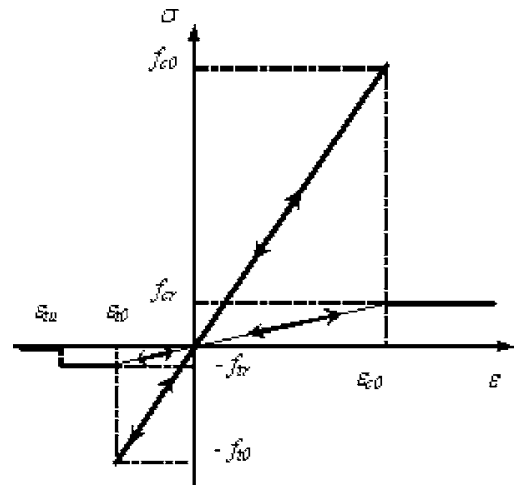

FIG. 4. Constitutive law for uniaxial compressive and tensile stress

presents an elastic-brittle damage constitutive relation with a given specific residual strength. The stiffness of elements degrades gradually as damage progresses, and the elastic modulus of damaged material can be defined as follows:

$$
E=(1-D) E_{0},
$$

where $D$ represents the damage variable, and $E$ and $E_{0}$ are elastic moduli of the damaged and the undamaged material, respectively.

The damage variable $D$ ranges from zero for the undamaged material to one for damaged material. With regard to the constitutive law shown in Fig. 4, the parameter $D$ can be calculated as

$$
D=\left\{\begin{array}{ll}
0 & \varepsilon>\varepsilon_{t 0} \\
1-\frac{f_{\mathrm{tr}}}{E_{0} \varepsilon} & \varepsilon_{\mathrm{tu}}<\varepsilon \leqslant \varepsilon_{t 0} \\
1 & \varepsilon \leqslant \varepsilon_{t 0}
\end{array},\right.
$$

where $f_{\text {tr }}$ is the residual tensile strength, and $E_{0}$ is the Young's modulus for undamaged elements. $\varepsilon_{t 0}$ is the strain at the elastic limit, which is the so-called threshold strain for tensile damage, while $\varepsilon_{\mathrm{tu}}$ is the ultimate tensile strain, at which the element would be completely damaged in tensile mode.

Additionally, we assume that the damage of mesoscopic element in multiaxial stress fields is also isotropic elastic. According to the method of extending one-dimensional constitutive laws under uniaxial tensile stress to complex tensile stress conditions, which was proposed by Mazars and Pijaudier-Cabot [25] for a constitutive law of elastic damage, we can easily extend the constitutive law described above to a three-dimensional stress state.

It must be emphasized that, when $D=1$, the damaged elastic modulus is zero, which may cause the finite element analysis to halt. Therefore, a relatively small number, i.e., $10^{-5}$ is specified for the limit elastic modulus.

The above constitutive law only considers the situation when element is damaged in tensile failure mode. But compressive or shear failure mode also occurs when the elements subjected to high compressive or shear stresses; thereafter shear damage at element scale level is also considered in our study for elements under compressive or shear stresses. The
Mohr-Coulomb criterion, as expressed in Eq. (3), is selected to be the second damage threshold

$$
F=\sigma_{1}-\frac{1+\sin \phi}{1-\sin \phi} \sigma_{3} \geqslant f_{c 0},
$$

where $\sigma_{1}$ and $\sigma_{3}$ are the maximum and minimum principal stresses, respectively. $f_{c 0}$ is the uniaxial compressive strength and $\varphi$ is the internal friction angle of this element.

With regard to the constitutive law in Fig. 4 when the element is damaged in shear mode, the damage variable $D$ can be described as follows:

$$
D=\left\{\begin{array}{ll}
0 & \varepsilon<\varepsilon_{c 0} \\
1-\frac{\lambda \varepsilon_{c 0}}{\varepsilon} & \varepsilon \geqslant \varepsilon_{c 0}
\end{array},\right.
$$

where $\varepsilon_{c 0}$ is the strain at the peak compressive principal stress under uniaxial compressive stress state, and $\lambda$ is the coefficient of residual strength.

The mechanical behavior of quasibrittle materials under multiaxial compression is mainly characterized by a considerable increase of strength and prepeak strain at high confinement level. When an element is under multiaxial stress state and its stress condition satisfies the Mohr-Coulomb criterion, shear damage occurs, and we must consider the effect of other principal stresses in this model during damage evolution process.

When the Mohr-Coulomb criterion is satisfied, we can calculate the minimum principal strain (maximum compressive principal strain) $\varepsilon_{c 0}$ at the peak value of maximum principal stress (maximum compressive principal stress)

$$
\varepsilon_{c 0}=\frac{1}{E_{0}}\left[f_{c 0}+\frac{1+\sin \phi}{1-\sin \phi} \sigma_{3}-\mu\left(\sigma_{1}+\sigma_{2}\right)\right] .
$$

In this respect, we assume that the shear damage evolution is only related to the maximum compressive principal strain $\varepsilon_{1}$. So, we use the maximum compressive principal strain $\varepsilon_{1}$ of damaged element to substitute the uniaxial compressive strain in Eq. (3).

From the above expression of damage variable $D$, which is generally called damage evolution law in damage mechanics, together with the Eq. (2), we can calculate the damaged elastic modulus of the element at each stress or strain level. For simplification, the Poisson's ratio of the damaged element is assumed to be increased by certain value but independent of the stress states and damage evolution process.

In this model, the element may gradually damage according to the above elastic damage constitutive law. Only elements whose ultimate tensile strain has been attained are displayed as fractures with black color in the postprocessing figures. Both tensile damage and shear damage leads to the mechanical property degradation of elements, but tensile damage is considered to be the direct cause of fracture initiation. In this respect, the initiation, propagation and interaction of multiple fractures is easily simulated.

We use a newly developed three-dimensional finite element code named RFPA $^{\mathrm{D}}$-PARALLEL (rock failure process analysis code for parallel computation) to solve the problem 
[27]. A parallel computer with 64-CPUs was used to fulfill the task.

The stretching of the layered model corresponds then to a gradual, homogeneous change of coordinates. Of interest are the ensuing pattern of fractures and the dependence of the pattern on the stretching in terms of principal stress ratio. We use a constant displacement increment in the $x$ direction along the left and right boundaries, and use another increment in the $y$ direction along the top and bottom boundaries. We define $\lambda$ as the loading ratio of the displacement in the $x$ direction to the displacement in the $y$ direction in order to model various principal stress ratios. We select $\lambda=0,0.125$, $0.25,0.375,0.5,0.625,0.75,0.875$, and 1 in our modeling, representing loading from directional to isotropic conditions.

During the modeling, one step of the calculation involves the computation of the forces acting on the elements and reduction of the mechanical properties of those elements that fail (because their strength is smaller than the acting stress). Iterating the procedure leads to fracture propagation.

Our method is most closely related to that of Hornig et al. [26], in that it treats materials as heterogeneous and the fractures are modeled by breaking individual lattice spring that reaches its strength. Hornig et al. [26] modeled the coating through an array of springs and account for its statistical inhomogeneities by assigning each spring a breakdown threshold taken from a given probability distribution. The coating breaks under a quasistatical, slowly increasing strain. In lattice model, breaking a lattice spring means removing it from the lattice. In contrast to their work, however, we treat the failed elements in different way. Instead, removing the failed element from the model, we replace the element with a very low Young's modulus. It is important to mention that when the compressive strain in the failed element exceeds certain strain, the recompaction behavior occurs and the stiffness of the failed element may restore to resist the continuous compression.

\section{SIMULATION RESULTS}

Figure 5 shows the numerically obtained images that demonstrate the complete process of the fracture pattern evolution for isotropic loading $(\lambda=1)$. We took a bird's eye view over the central cross-section cut from the embedded layer. It can be seen from Fig. 5(a) that in the first stage of fracture pattern development, fractures nucleate at a small number of points. Defects (model elements with lower strength) in the layer structure presumably serve as nucleation sites. We found that fractures tend to nucleate at the weaker sites and in many cases do not propagate long distances across the layer, but rather move in small steps from one weak site to the next, occasionally meeting another fracture moving in a similar fashion [Figs. 5(b) and 5(c)]. After the initiation of a few longer fractures, most new fractures start at the vicinity of existing fractures and propagate away from their parent fracture, approximately at right angles. Finally, successive generations of fractures form, mostly joining older fractures and forming a complicated array of polygons [Figs. 5(c) and $5(\mathrm{~d})]$.

As predicted by fracture spacing theory $[10,14]$, numerical simulations of the isotropic fracture system show a com- mon process, called fracture infilling. As shown in Fig. 5(e), a new fracture propagates away from an existing fracture, and eventually stops when it runs into another fracture. The region is then fragmented into two parts. Although randomly distributed, the size of the polygonal fractured area is found to be approximately the same with some deviation that should be the outcome of the heterogeneity existed in the layer. It is very interesting to note that after reaching a certain strain, the number of polygons formed in the fractured layer no longer increases [Fig. 5(e)]. Instead, interface debonding is found to dominate the postfailure processes [Fig. $5(\mathrm{f})]$. This suggests that fracture saturation also exists in polygonal fracture pattern. However, the role of accommodation of further strain may not only play by existing vertical fractures as predicted in two-dimensional model, but also by interfacing fracturing, which will be discussed later.

In order to understand the contribution of stress distribution to find the evidence for fracture saturation, we plot the minimum principal stress distribution in the right column of Fig. 5. It is shown that, network fractures behave as free surfaces, and as a result the normal tensile stress in the vicinity of a fracture is greatly reduced and, particularly, minimum tensile stresses in the center of polygonal shaped islands are found to be very small when fracture saturation is well developed [Fig. 5(e)], and therefore the formation of new fractures is inhabited. This zone of reduced stress, referred to as the stress reduction shadow, with the tractionfree islands, scales with layer thickness and is responsible for the observed correlation between fracture spacing and layer thickness $[1,28]$.

Figure 6 shows the numerically obtained fracture patterns well developed in the central cross section of the embedded fractured layer for the nine models, corresponding to the loading conditions for $\lambda=0,0.125,0.25,0.375,0.5,0.625$, $0.75,0.875$, and 1 (supplementary images and movies are placed at EPAPS electronic depository, i.e., see Ref. [29]). It is important to note that the modeling results indicate a continuous pattern transition from parallel fractures to polygonal fractures, depending on the loading conditions in terms of principal stress ratio. In contrast to the results obtained from $\lambda=1$ (Fig. 5), Fig. 6(a) for model subjected to directional loading $(\lambda=0)$ shows an obvious parallel fracture pattern. Due to the effect of heterogeneity, the obtained fracture propagations demonstrate a more realistic pathway. Most of the fractures terminated at certain lengths because they overlapped other fractures propagating from the opposite edge or interior. Even for those that appear to extend from one edge to the other actually are composed of several closely-spaced echelon segments. This fracture pattern resembles very well with that observed in experiments by Wu and Pollard using a poly(methylmethacrylate) (PMMA) sample of surface coating [10].

In the biaxial loading with principal stress ratio $\lambda$ ranging from 0.125 to 0.5 , another dominated mode of pattern formation is observed, shown in Figs. 6(c)-6(e), particularly for $\lambda=0.375$; Shorlin et al. [28] refer this process as laddering in their experiments of shrinkage fracture modeling when a thin layer of alumina/water slurry dries. Figure 6(d) shows that, during the fracture forming process, fractures which were perpendicular to the parallel fractures were formed and they 


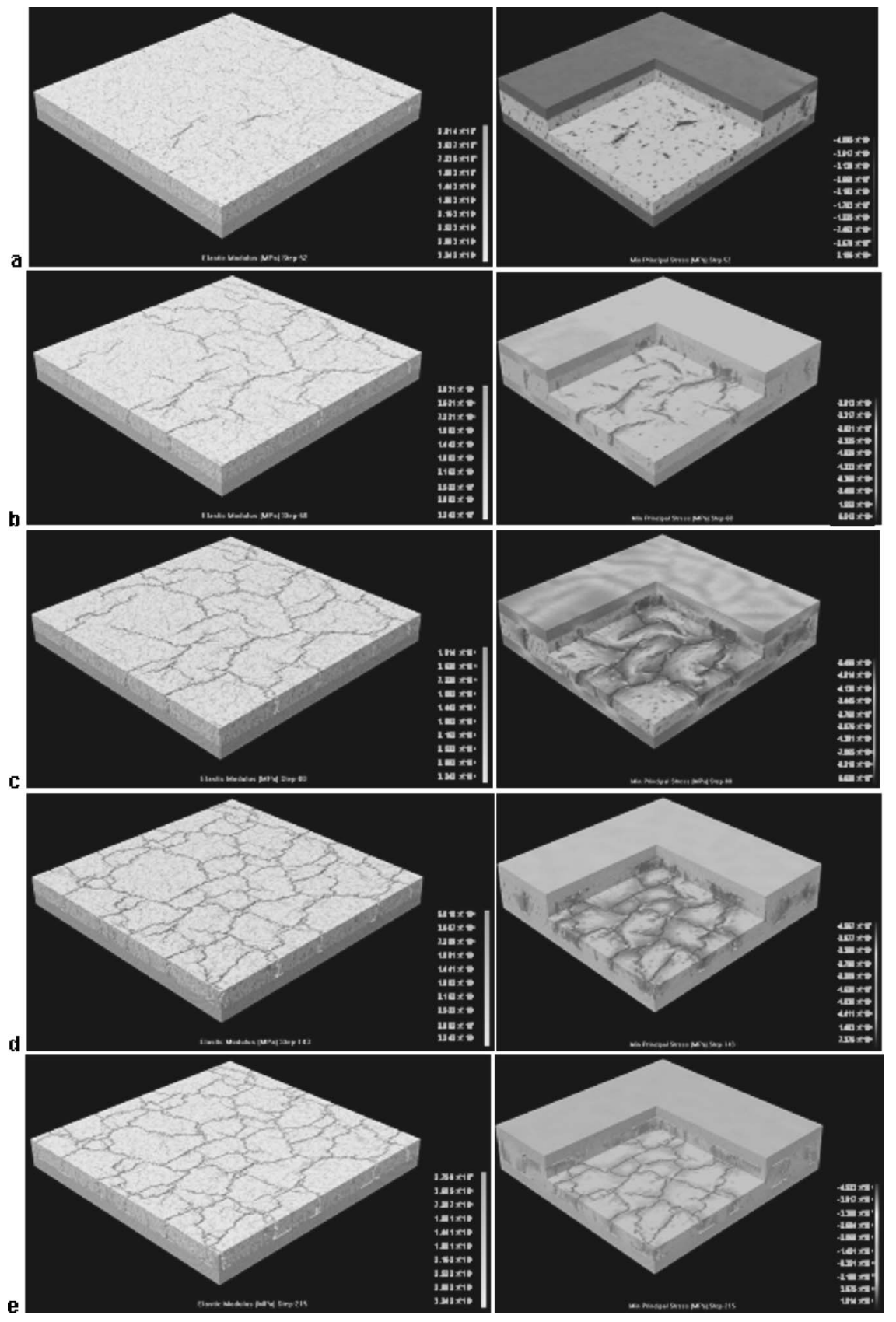

FIG. 5. RFPA modeling results of fracture evolution (left column) and induced stress redistribution (right column) for model with principal stress ratio $\lambda=1$. The cross sections are taken from the central plane in the embedded layer. The stress is expressed in minimum principal stress. (a)-(f) represent the different characteristic stages of the fracture process.

are like the rungs of a ladder. The ordering seen in Figs. 6(c)-6(e) is not so regular as ladders, as observed in experiments made by Shorlin et al. [28]. This is presumably because of the stress being built up homogenously across the whole sample at once.

Our modeling technique provides a valuable insight concerning fracture processes that are difficult, if not impossible, to observe in nature and difficult to consider using stress analysis approaches. Particularly the three-dimensional modeling with the fracture approach has shed considerable light on fracture pattern development in layered materials. As mentioned above, most theoretical models in the literature that explain fracture spacing are from the plane-strain perspective of a vertical, bedding-perpendicular cross section, where the fracture length is assumed to be infinite, and the only variable fracture dimensions are layer height and frac- ture opening [1,5,14,29-31]. The modeling approach described here reveals that the formation of fracture patterns is strongly influenced by loading conditions and the fracture spacing under plane-strain conditions can only have limited role on the understanding to the problem.

Fractures develop in the embedded layer when the stretch of the layers induces sufficient stress that the layer fractures. Our modeling indicates that, under isotropic stretch conditions, isolated fractures initially appear by nucleation at a few points. Triplet junctions, at which three fractures meet with junction angles of $120^{\circ}$, are formed by such nucleation in the early stages of pattern development. At later times, however, fractures meet predominately at $90^{\circ}$ junctions. As explained by Shorlin et al. [28], this is due to the fact that fractures propagate in the direction which most efficiently relieves the stress. Since the stress near a given fracture is 


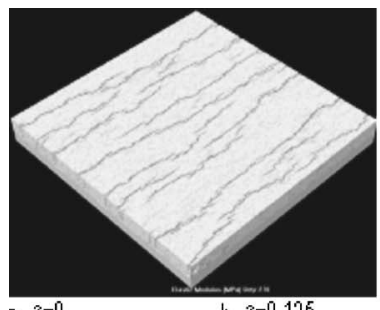

a. $x=0$

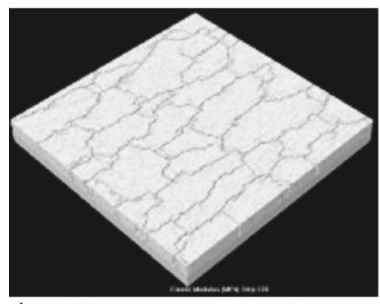

d. $x=0.375$

e. $x=0.5$

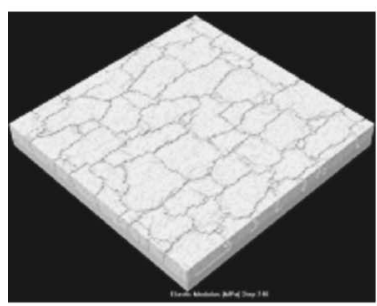

g. $\lambda=0.75$

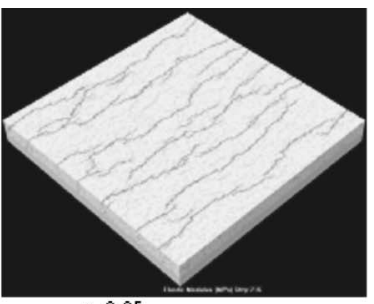

c. $\lambda=0.25$

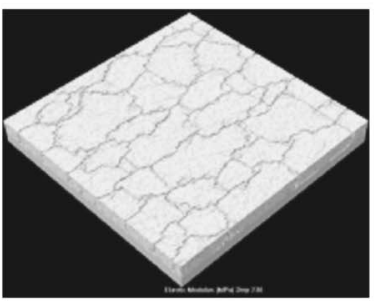

f. $x=0.625$

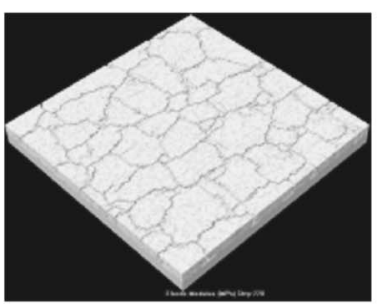

i. $\lambda=1$
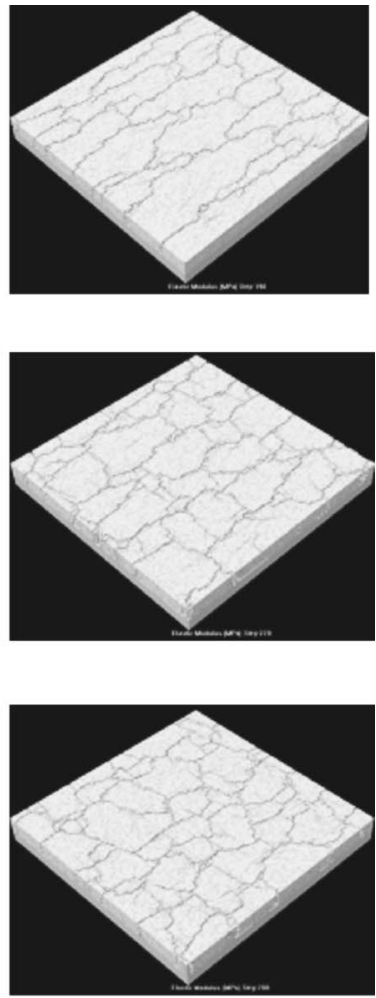

FIG. 6. RFPA modeling results for models with different principal stress ratio. Left: fracture patterns; Right: fracture locations. (a)-(i) corresponding to the principal stress ratio $\lambda=0,0.125,0.25$, $0.375,0.5,0.625,0.75,0.875$ and 1 , representing far-field loading conditions from directional loading to isotropic loading (see Ref. [29]). parallel to its surface, other fractures will tend to approach and meet it at right angles [28]. However, the abovementioned situation is only observed in the model under isotropic stretch or with principal stress ratio, $\lambda$, close to 1 . From the numerical simulations of the models with different principal stress ratio, $\lambda$, we also observed a transition in the distribution of junction angles as the ratio, $\lambda$, was decreased: for $\lambda$ less than 0.5 , we observed a marked increase in the number of junctions larger than $120^{\circ}$ or less than $60^{\circ}$. In the extreme situation, that is, directional stretch, the junction angle becomes $180^{\circ}$ or zero, and the parallel fractures dominate.

Interface debonding is found to be dominating the fracture development upon reaching the fracture saturation stage. Before that, mechanically the fractures arrest at the layer boundary during sequential infilling. Only after the fracture saturation is reached, the interface debonding dominates fracture occurrence. As an example, Fig. 7(a) show the modeling results of interface debonding for model under isotropic loading $(\lambda=1)$. During the fracture infilling process [as shown in Figs. 5(a)-5(e)], polygonal fracture network forms gradually until the fracture saturation is reached [Fig. 5(f)]. After that, as shown in Fig. 7(a), the interface debonding starts from the fracture network, which forms many "islands" (the areas bonded to the top layer) and "lake" (the area of interface debonding) surrounding them. When external loads continue to increase, debonding area increases. Consequently, the islands become smaller and smaller, whereas the lake around the islands become larger and larger. The previous theory about fracture spacing using two-dimensional and plane-strain models suggests that further strain after the fracture saturation will be accommodated by the opening of the existing fractures. Here, our three-dimensional modeling provides a second mechanism for this strain accommodation, that is, the fractures propagation along the layer interface.

Looking at the fracture induced stress redistribution in the interface area after fracture saturation helps to illustrate the interface debonding mechanism. As sown in Fig. 7(b), comparing with other areas, the tensile stresses in the islands may

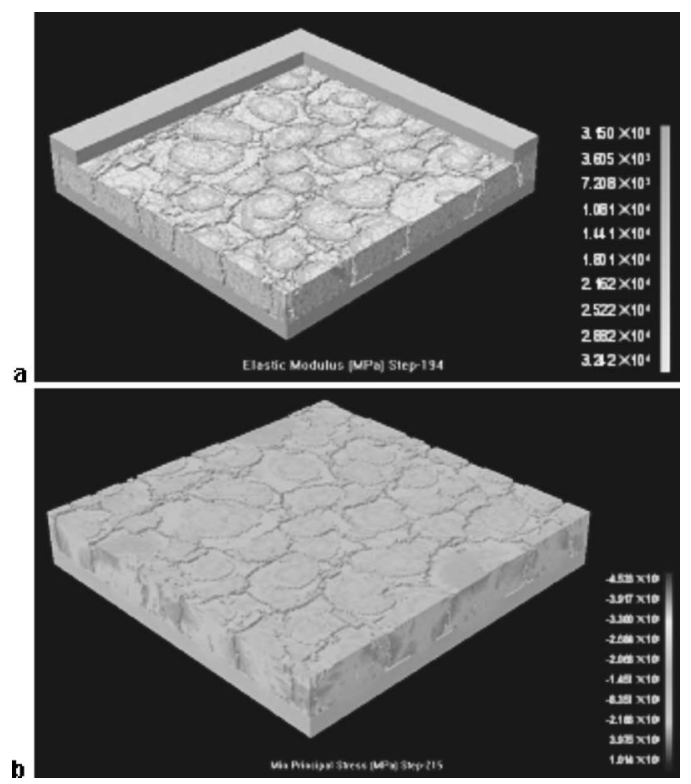

FIG. 7. RFPA modeling results of interface fracturing. induced stress redistribution for model with principal stress ratio $\lambda=1$. The cross sections are taken from the interface plane: (a) the interface debonding; (b) stress redistribution. The stress is expressed in minimum principal stress. 
be high enough to cause further debonding of the interface, and, therefore, the fractures propagating horizontally in the layer interfaces dominate the fracture pattern development.

\section{DISCUSSIONS}

In our numerical investigations, three types of numerical tests were performed: "isotropic stretch," in which the entire layer was stretched uniformly with principal stress ratio $\lambda$ $=1$; "anisotropic stretch," in which the layer was stretched biaxially with principal stress ratio $0<\lambda<1$ and "directional stretch," in which the layer was loaded from two ends, that is, uniaxially with principal stress ratio $\lambda=0$. We observed a change in the way in which fracture occurred as the loading method in the system was varied: for isotropic stretch, more regular shaped polygons formed. For anisotropic loading, polygons did not formed in regular shape, but rather formed by laddering. In this process, fractures propagate more or less parallel to each other. As the stretch strain increased further, perpendicular fractures form, joining two of the parallel fractures like the rungs of a ladder. The uniaxial stretch produces the pattern of parallel fractures.

The modeling results that fracture set reaches saturation with respect to principal stress ratio has important implications for the interpretation of fracture spacing data. It is generally recognized from fracture spacing theory $[10,14]$ that fractures in an embedded layer under uniaxial stretch usually tend to distribute approximately uniformly when fracture set is well developed (saturation), and it has been used as a basic assumption in both empirical and analytical models of fracture spacing. It is also said that a low deviation of fracture spacing represents a high degree of fracture saturation. Our modeling shows that this principle is no longer valid for situations that three-dimensional effects and multiaxial loading conditions have to be considered. It is well known from empirical facts that, in some cases, highly scattered fracture spacing is observed but no satisfactory explanation has been put forward as to why this scatter exists. Some investigators believed heterogeneity may serve as a possible reason, but the fracture pattern transition from parallel to polygonal implies that heterogeneity is not the only reason, particularly in the case for isotropic loading conditions. We use Fig. 8 to illustrate this point. The common method for measuring fracture spacing is the line (or scanline) method [10] (Fig. 8). Traverses are taken perpendicular to the average strike of the fracture set either along cross sections of a layer or along the surface of a layer to estimate the distance, $L$, between two neighboring fractures. The fracture spacing theory $[10,14]$ indicates a consistent measure of $L$ for a well-developed fracture pattern (saturated). However, this is true only when the layer is loaded uniaxially, in which parallel fractures forms. For fractured layer under biaxial loading conditions, the line spacing can be widely scattered even for a welldeveloped fracture pattern (Fig. 6). As shown in Fig. 8, even for isotropic loading $(\lambda=1)$, due to the island fracture network developed in $x-y$ plane, the spacing observed in two scanlines perpendicular to each other across the central point shows very different fracture distributions. When we plotted the spacing $L$ versus the principal stress ratio $\lambda$ for data

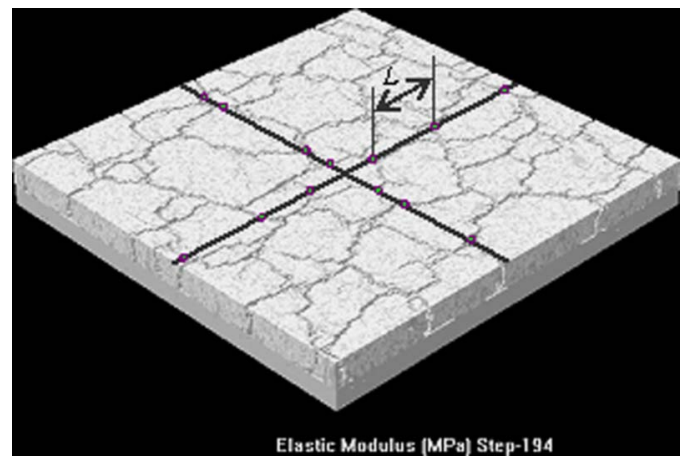

FIG. 8. (Color online) Measurement of fracture spacing using scanlines (red) across the central point of the numerical model and parallel to two principal stress directions, respectively. The points in the lines indicate the intersect point of line with fractures. The distance $L$ between two neighboring points represents its fracture spacing.

obtained using such scanline method [Fig. 9(a)], we found that the fracture spacing measured from lines with different directions show a different scale law of fracture spacing to principal stress ratio. When the ratio increases from 0 to 1 , the spacing $L y$ measured in the scanline parallel to the minimum principal stress decreases, whereas the spacing $L x$ measured in the scanline parallel to the maximum principal stress shows a very little increase. In both situations, big scatter is founded. Bearing this in mind, it is reasonable to raise doubts about the usefulness of field data taken along scanlines on cross sections of a layer. If we are correct, the suitability of the widely used scanline method for measuring fracture spacing [10] should be carefully reconsidered. Although an alternative area method was suggested by $\mathrm{Wu}$ and Pollard [10], their method seems not suitable for fracture patterns such as the polygonlike fracture network. Further work on a more general method for measuring fracture spacing, which is useful for outcrop studies, is worthwhile to pursue. Since the polygonal area statistically shows a uniform distribution when the fractured layer is well developed, a scale for fracture network area spacing with layer thickness may provide an alternative measure for fracture spacing investigation. Although further field evidence is needed to support our observation, we strongly believe that, this fact, along with the errors introduced by the spacing measurement method, could explain more reasonably the considerable scatter in some field data reported in the literature. This conclusion also supports the point made by $\mathrm{Wu}$ and Pollard [10] that one of the items to consider when gathering spacing data is how well the fracture set is developed. Measuring fracture spacing using the scanline method from fracture sets without any information about their loading conditions is unlikely to lead to meaningful insights about the fracture spacing, because spacing is so sensitive to the applied principal stress ratios.

\section{CONCLUSIONS}

Three major results are relevant to the study of fracture spacing.

First, numerical results of a continuous transition from parallel to polygonal fracture patterns with principal stress 

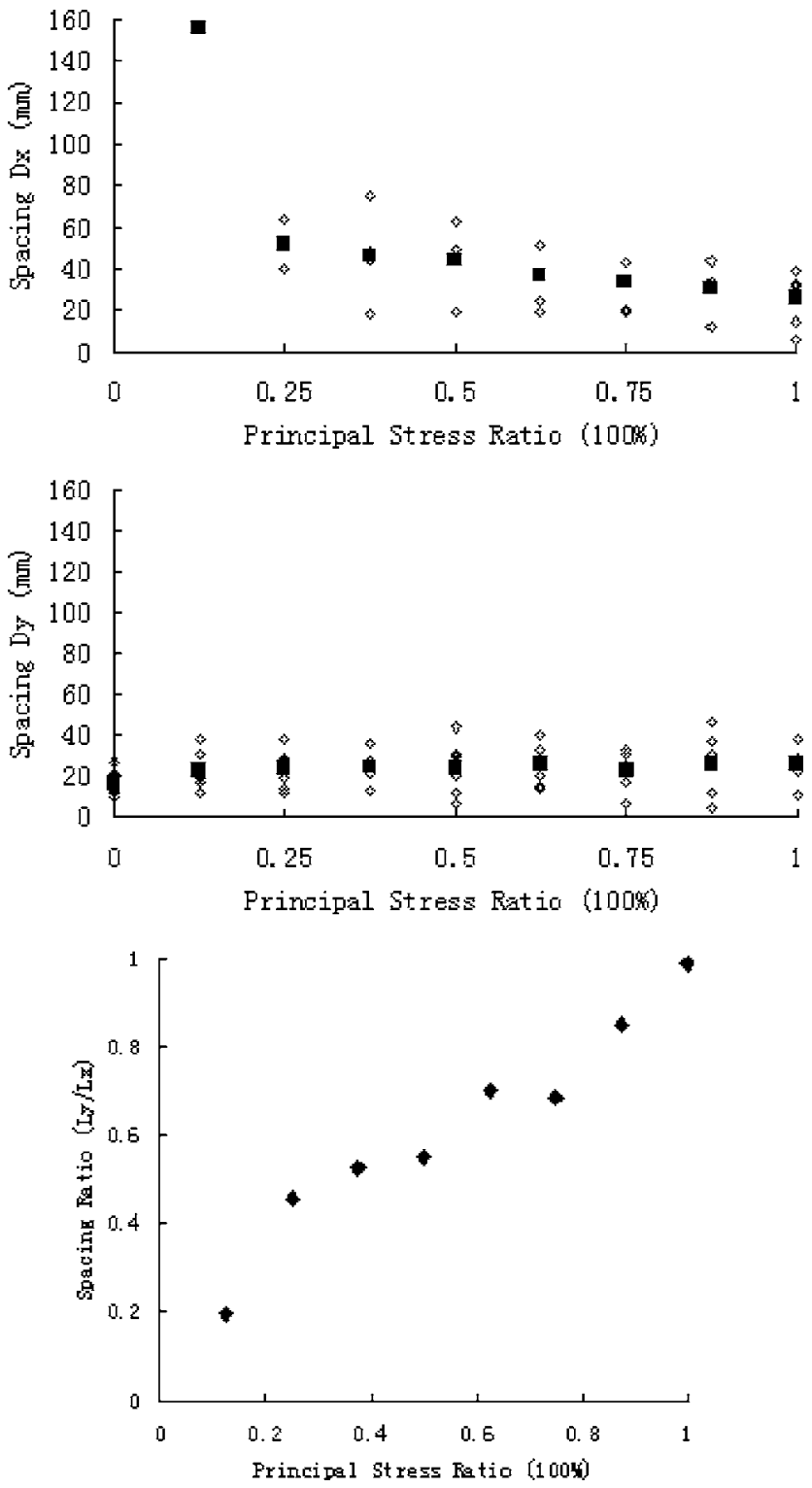

FIG. 9. RFPA modeling results of fracture spacing $L$ vs principal stress ratio $\lambda$. (a) Spacing $L x$ and $L y$ vs principal stress ratio $\lambda$. (b) Spacing $L y / L x$ vs principal stress ratio $\lambda$.

ratio provides the clear convincing theoretical explanation for fracture spacing. In our numerical investigations, three types of numerical tests were performed: isotropic stretch, anisotropic stretch, and directional stretch. We observed a change in the way in which fracture occurred as the loading method in the system was varied: for isotropic stretch, more regular shaped polygons formed. For anisotropic loading, polygons did not formed in regular shape, but rather formed by laddering. The uniaxial stretch produces the pattern of parallel fractures. If we define fracture spacing in a more general way, such as area spacing instead of line spacing, then, the fracture spacing law founded in the twodimensional plane strain model is also valid for the threedimensional model under biaxial loading conditions. That is, for any combination of layer-parallel principal stresses, fracture spacing decreases rapidly with strain in the early stages of loading and then decreases less rapidly, finally reaching a nearly constant value, beyond which a greater applied strain will not change the spacing significantly.

Second, the modeling shows how subcritical fracture initiation, growth, and coalescence can be used to predict cluster formation, a phenomena representing an exception to the widely accepted fracture spacing theory $[10,14]$ based on models assumed to be homogeneous, in which the patterns are formed by fractures that propagate in straight lines and interact to form an array of polygons. Our models with heterogeneity being taken into account show that the fractures tended to propagate shorter distances, and the polygons are formed by the coalescence of fractures.

Third, interface debonding is found to be dominating the fracture development upon reaching the fracture saturation stage. Before that, mechanically the fractures arrest at the layer boundary during sequential infilling. Only after the fracture saturation is reached, the interface debonding dominates fractures occurrence. The previous theory about fracture spacing using two-dimensional and plane-strain models suggests that further strain after the fracture saturation will be accommodated by the opening of the existing fractures. Our three-dimensional modeling provides a second mechanism for this strain accommodation, that is, the fractures propagation along the layer interface.

In summary, we have performed an extensive numerical modeling study of fracture pattern formation in cases from the directional to isotropic stretch. Our results provide reality modeling of the experimentally observed phenomena, which helps the understanding of the complexity of the pattern formation process in this system.

\section{ACKNOWLEDGMENTS}

This work was supported by NSFC of China and ChinaGrid Program. We thank Professor Stephen Morris for his valuable suggestions to improve the paper.
[1] D. W. Hobbs, Sci. News (Washington, D. C.) 104, 550 (1967).

[2] F. L. Ladeira and N. J. Price, J. Struct. Geol. 3, 179 (1981).

[3] Z. P. Bazant, Appl. Mech. Rev. 39, 675 (1986).

[4] Q. Huang and J. Angelier, Geol. Mag. 126, 355 (1989).

[5] W. Narr and J. Suppe, J. Struct. Geol. 13, 1037 (1991).

[6] T. Rives, M. Razack, J. P. Petit, and K. D. Rawnsley, J. Struct. Geol. 14, 925 (1992).
[7] M. D. Thouless, E. Olsson, and A. Gupta, Acta Metall. Mater. 40, 1287 (1992).

[8] H. Colina, L. Arcangelis, and S. Roux, Phys. Rev. B 48, 3666 (1993).

[9] M. R. Gross, J. Struct. Geol. 15, 737 (1993).

[10] H. Q. Wu and D. D. Pollard, J. Struct. Geol. 17, 887 (1995).

[11] A. Becker and M. R. Gross, Tectonophysics 257, 223 (1996). 
[12] C. Pascal, J. Angelier, and M. C. Cacas, J. Struct. Geol. 19, 1273 (1997).

[13] S. C. Ji and K. A. Saruwatari, J. Struct. Geol. 20, 1495 (1998).

[14] T. Bai, D. D. Pollard, and H. Gao, Nature (London) 403, 753 (2000).

[15] D. H. Timm, B. B. Guzina, and V. R. Voller, Int. J. Solids Struct. 40, 125 (2003).

[16] K. M. Crosby and R. M. Bradley, Phys. Rev. E 55, 6084 (1997).

[17] S. Krishnamurthy and I. Reimanis, Surf. Coat. Technol. 192, 291 (2005).

[18] M. P. Fischer, M. R. Gross, T. Engelder, and R. J. Greenfield, Tectonophysics 247, 49 (1995).

[19] K. L. Wang, J. F. Cassidy, I. Wada, and A. J. Smith, Geophys. Res. Lett. 31, L01605 (2004).

[20] R. Gabrielli and G. Caldarelli, Europhys. Lett. 45, 13 (1999).

[21] O. Ronsin and B. Perrin, Europhys. Lett. 38, 435 (1997).

[22] T. Boeck, H. A. Bahe, S. Lampenscherf, and U. Bahr, Phys. Rev. E 59, 1408 (1999).

[23] C. A. Tang, Railw. Age 34, 249 (1997).
[24] W. C. Zhu and C. A. Tang, Acta Med. Austriaca Suppl. 37, 25 (2004).

[25] J. Mazars and G. Pijaudier-Cabot, J. Eng. Mech. 115, 345 (1987)

[26] T. Hornig, I. M. Sokolov, and A. Blumen, Phys. Rev. E 54, 4293 (1996)

[27] Y. B. Zhang, C. A. Tang, Z. Z. Liang, T. H. Ma, and H. Zhang, Chin. J. Rock Mech. Eng. (to be published).

[28] K. A. Shorlin, J. R. Bruyn, M. Graham, and S. W. Morris, Phys. Rev. E 61, 6950 (2000).

[29] See EPAPS Document No. E-PLEEE8-73-185605 for supplementary images and movies showing a continuous pattern transition from parallel fractures to polygonal fractures. For more information on EPAPS, see http://www.aip.org/pubservs/ epaps.html

[30] D. D. Pollard and P. Segall, in Fracture Mechanics of Rock, edited by B. K Atkinson (Academic Press London, 1987), pp. 277-349.

[31] J. E. Olson, Geol. Soc. Spec. Publ., London, 231, 73 (2004). 